Proceedings of the 2012 Winter Simulation Conference

C. Laroque, J. Himmelspach, R. Pasupathy, O. Rose, and A.M. Uhrmacher, eds.

\title{
ASSESSMENT METHODOLOGY FOR VALIDATION OF VEHICLE DYNAMICS SIMULATIONS USING DOUBLE LANE CHANGE MANEUVER
}

\author{
Emir Kutluay \\ Technische Universität Darmstadt \\ Petersen Strasse 30 \\ D-64287 Darmstadt, GERMANY
}

\author{
Hermann Winner \\ Technische Universität Darmstadt \\ Petersen Strasse 30 \\ D-64287 Darmstadt, GERMANY
}

\begin{abstract}
The simulation of vehicle dynamics has a wide array of applications in the development of vehicle technologies. This study deals with the methodological aspect of the problem of assessing the validity of a simulation using double lane change maneuver as the experimental data source. The maneuver time history is analyzed. Problems in handling the obtained measurements and possibilities to assess the maneuver are examined. Techniques to split and align the data are presented and compared. Methodologies to handle the experimental and simulation data are introduced. The presented methods can be utilized in order to achieve more time and cost efficient simulation projects with increased model confidence.
\end{abstract}

\section{INTRODUCTION}

Computer simulation models are utilized in nearly every research and product development process in automotive industry. One of these utilization fields is prediction of the dynamic response of existing or proposed vehicle designs. The simulation of vehicle dynamics has a wide array of applications in the development of vehicle technologies, i.e. active suspensions, chassis design, controller design, driver assistance systems, development of simulators for ergonomics research, etc. Although simulation environments, measurement tools and mathematical theories on vehicle dynamics are well established, the methodical link between the experimental test data and validity analysis of the simulation model is still lacking.

Comparing the results of the simulation models against that of the real world measurements, be it long term measurements on public roads to determine tire wear (Braghin et al. 2006) or execution of standardized maneuvers on proving grounds (Allen et al. 1992; Ozan et al. 2010), is the standard application for validation of vehicle dynamics simulations. The maneuvers that are utilized in these experiments are usually the same maneuvers that are used to assess the dynamic properties of vehicle designs. The difference in validation studies is that the design in question is not the vehicle but the simulation model.

According to the scope of application, these maneuvers can be classified in two groups, fundamental maneuvers and purpose dependent maneuvers. The fundamental maneuvers are used to determine the main dynamical characteristics of the vehicle in time and frequency domain for steady state, transient, periodical and stochastic responses. Steady state cornering (ISO 2004), step response and sine sweep maneuvers (ISO 2003) are such examples. These manuvers are the maneuvers which clearly exhibit general dynamic characteristics of a vehicle, and are economically feasible, experimentally repeatable and consequentially comparable, but nonetheless in real life nonexistent. On the other hand, the purpose dependent maneuvers approximate a real life maneuver family. Power-off reaction of a vehicle in a turn (ISO 2006) is an example to this class of maneuvers.

Double lane change maneuver is one such maneuver. It approximates an emergency maneuver case where the vehicle must be steered to the adjacent lane and back. During such a maneuver an understeering, or oversteering, or even a rollover situation can occur. 


\section{Kutluay and Winner}

This study deals with the methodological aspect of the problem of assessing the validity of a simulation using double lane change maneuver as the experimental data source. The maneuver time history is analyzed. Problems in handling the obtained measurements and possibilities to assess the maneuver are examined. Techniques to split and align the data are presented and compared. Methodologies to handle the experimental and simulation data are introduced. The presented methods can be utilized in order to achieve more time and cost efficient simulation projects with increased model confidence.

\section{ANALYSIS OF THE MANEUVER}

\subsection{Double Lane Change Maneuver}

Double lane change maneuver approximates the behavior of a vehicle in the case where the driver needs to quickly switch from one lane to the other and back in the face of an emergency. During the maneuver the vehicle might understeer due to saturation tire forces in the front axle, or oversteer, especially during the counter steering phase, or even roll over because of the high lateral acceleration involved which occasionally happens with the vehicles with relatively higher center of gravity. The maneuver generally demonstrates the agility and capabilities of the vehicle in lateral dynamics.

Before ISO-3888 (ISO 1999) was issued; emergency lane change maneuver used to be simulated using an open loop sine steering input of one period length (ISO 1979; Winkler, Nisonger and Ervin 1978).

The amplitude of the wave affected the maximum lateral acceleration during the maneuver and decided the severity of the maneuver, typically ranging between $0.4 \mathrm{~Hz}$ and $1 \mathrm{~Hz}$ (ISO 1999, Heydinger et al. 1990). Typical metrics that were measured using this maneuver are:

- Time lags for the first and second half waves of the maneuver (using cross correlation)

- Ratio of the time lags

- Maximum output to maximum input ratios for half waves

- Ratio of the maximum output to maximum input ratios for half waves

These metrics are computed separately for each experimental run and then mean values and standard deviations are calculated. The state of the art standard used to simulate an emergency lane change is ISO3888/1. In this document, only test track is defined. That means the resulting maneuver is a closed loop maneuver, in which the test driver tries to follow the test track, contrary to its proposed forerunners which define only the shape of the steering input regardless of the track.

\subsection{Data Splitting}

The general time history of an emergency lane change maneuver, executed at $80 \mathrm{~km} / \mathrm{h}$ on a test track defined according to ISO-3888/1 shows that the trend of the input steering wheel angle is comprised of two distinct wave like motions: the first one is from when the vehicle leaves its original lane to when the vehicle reaches the second lane; the second one is from when the vehicle leaves the second lane to when the vehicle returns to its original lane.

Assuming that the velocity is held constant (depending on the aim of the experiment, speed drop can also be counted among possible performance metrics) the frequency and amplitude of these two motions should be very similar. However depending on the selected velocity value, the portion in the middle shows different characteristics as can be seen in Figure 1. Nevertheless, just like the open loop single sine input case, the time histories can be investigated in two portions. 


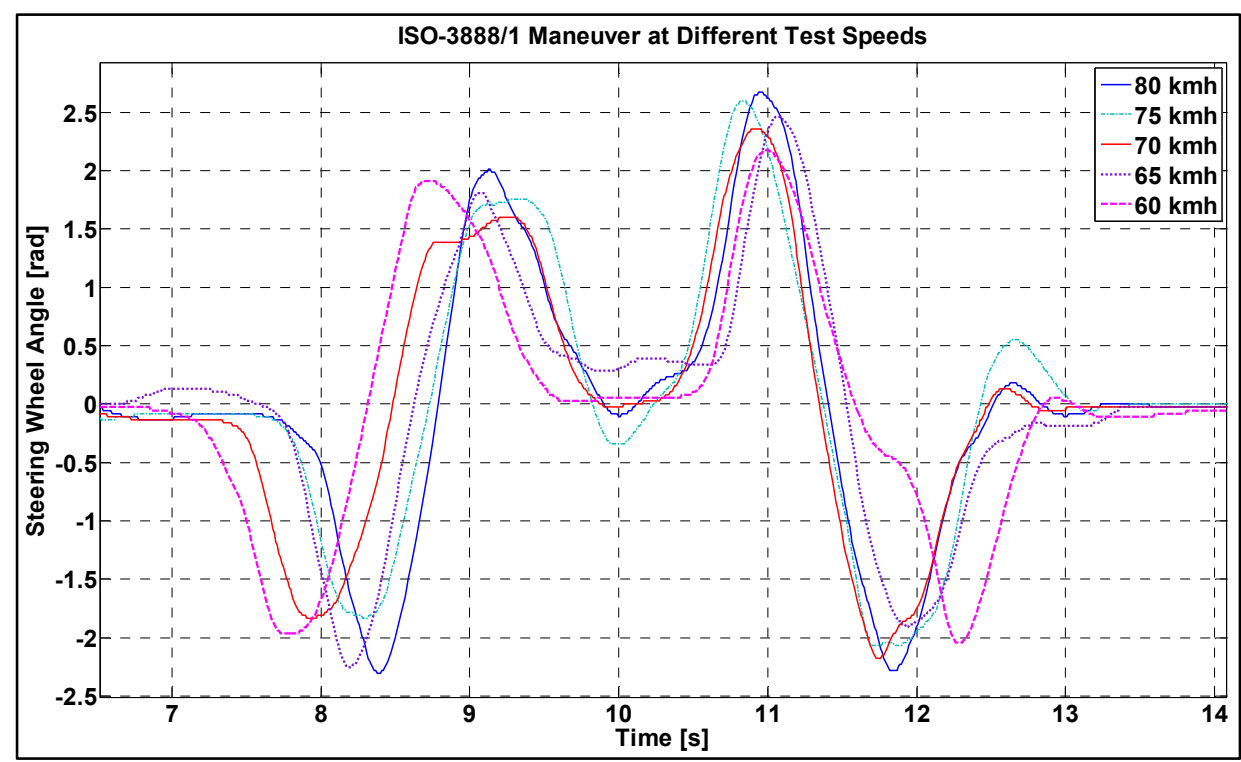

Figure 1: Time histories of double lane change maneuver for different test speeds

Depending on the test conditions (road, tire, vehicle type) and vehicle speed, the steering wheel angle can:

a. Reach a steady state in the middle portion of the maneuver (Figure 2).

b. Reach a local extremum in the middle portion of the maneuver (Figure 3).

c. Reach multiple local extrema in the middle portion of the maneuver, when the vehicle is in the second lane (Figure 4).

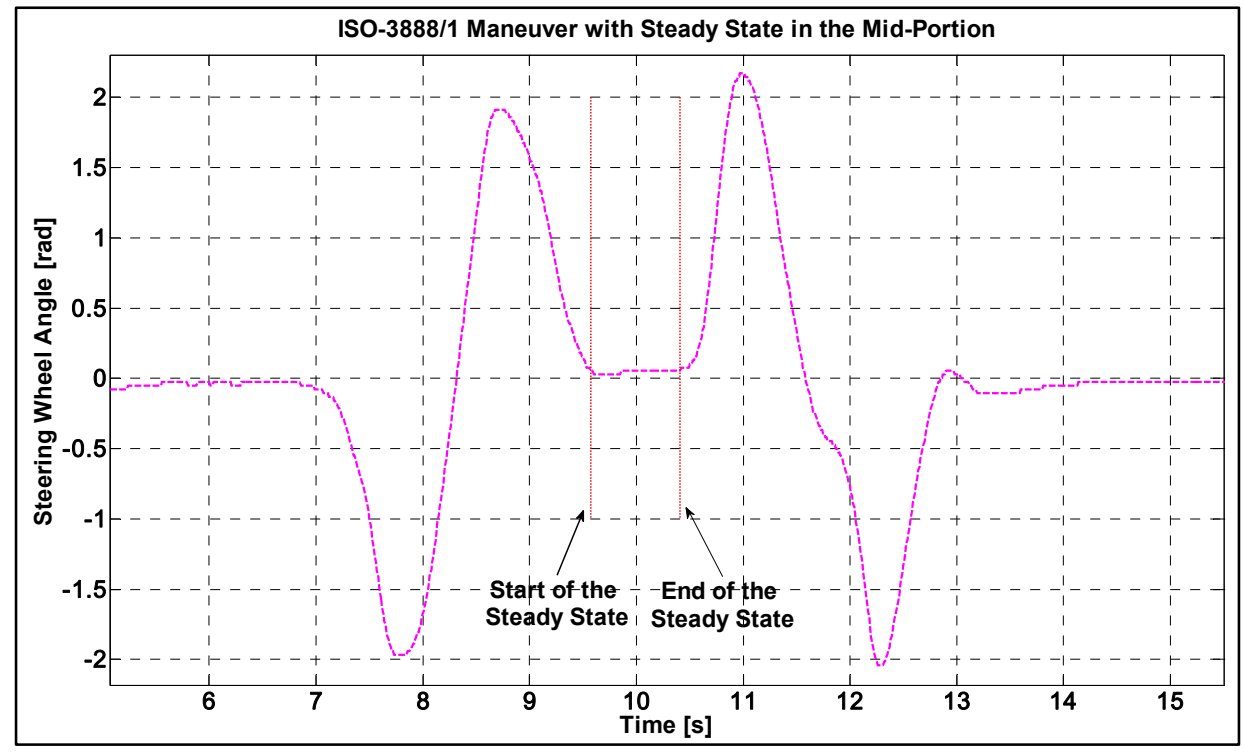

Figure 2: Double lane change maneuver with steady state in the mid-portion

According to these possibilities one can define the midpoint(s) relatively as:

a. Start and end of the steady state region, starting point being the end time for the first portion and end point being the start time for the second portion (Figure 2).

b. Where the single local extremum occurs (Figure 3). 


\section{Kutluay and Winner}

c. The first and the last local extremum, first being the end time for the first portion and last being the start time for the second portion (Figure 4).

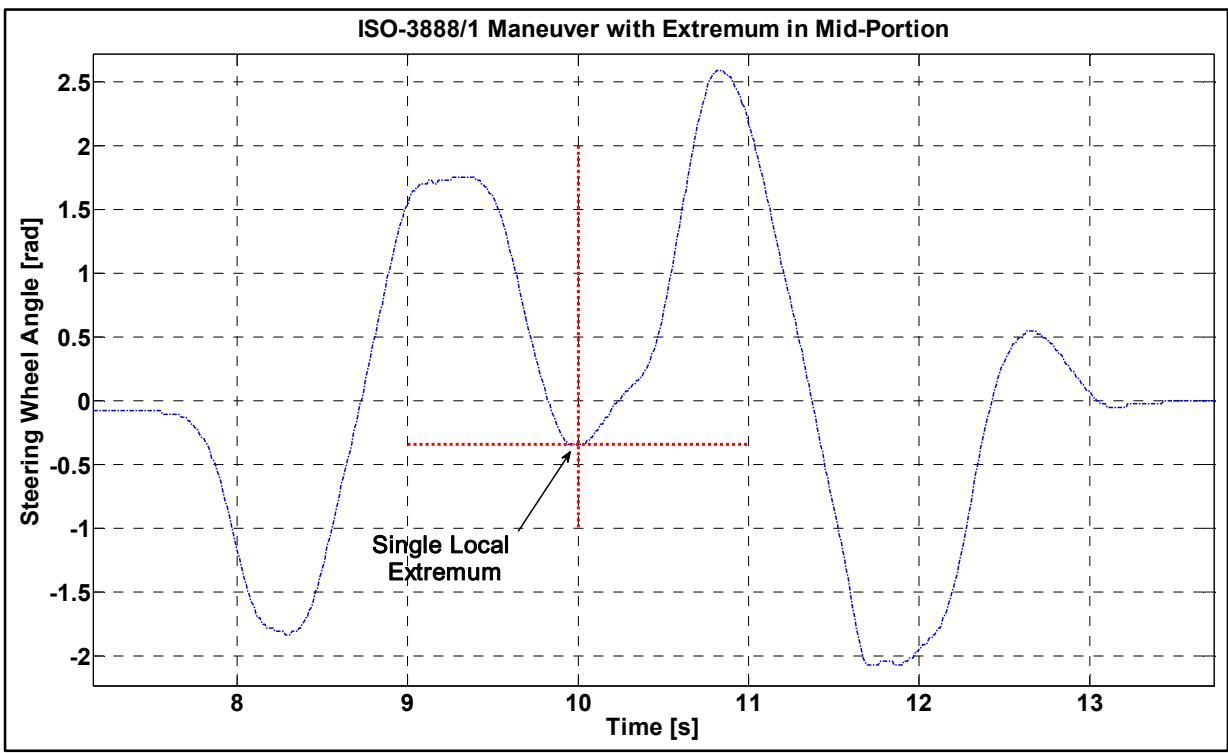

Figure 3: Double lane change maneuver with single local extremum in the mid-portion

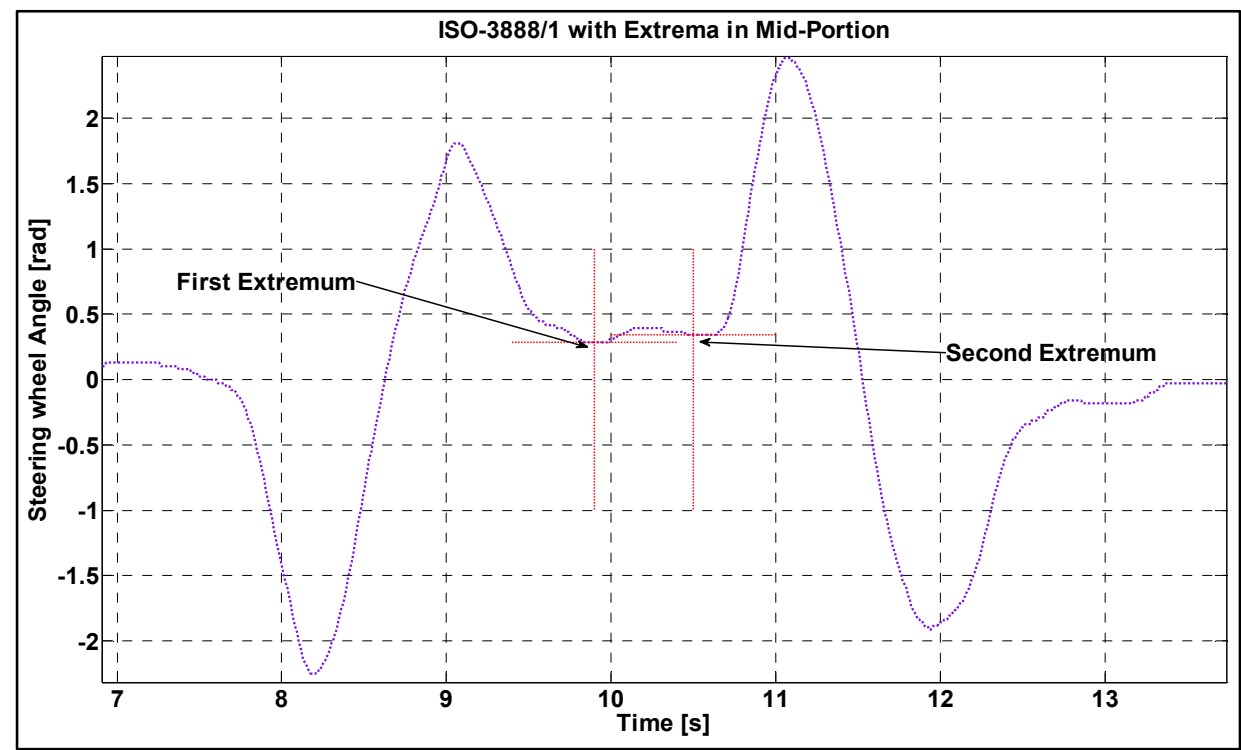

Figure 4: Double lane change maneuver with multiple local extrema in the mid-portion

All these three techniques are dependent on the conditions in the middle portion of the maneuver and the definition of the reference point(s) requires an experiment dependent approach. On the other hand only the number of experiment to experiment consistent characteristics is limited: entry straight driving, first sine-like input (with two extrema), second sine-like input (with two extrema) and exit straight line driving. Since the exit of the maneuver includes stabilizing the vehicle as the vehicle enters the exit lane, the entry straight driving is the only objective and experiment independent steady state property. Thus, if the reference point in the middle portion can be defined using this characteristic value together with one or more of the other experiment independent characteristic points, an objective and experiment independent definition of a reference point for data splitting can be reached. 


\section{Kutluay and Winner}

The proposed method to define the reference point requires three of the aforementioned characteristic values (Figure 5): steady state entry steering wheel angle (A), the last extremum of the steering wheel angle before the middle portion (B), the first extremum of the steering wheel angle after the middle portion (C).

- $50 \%$ descend time of the first input wave is defined using (A) and (B). This is the time when the steering angle reaches mean value of $(\mathrm{A})$ and $(\mathrm{B})$ before the intermediate portion.

- $50 \%$ ascend time of the second wave is defined using (A) and (C). This is the time when the steering angle reaches mean value of $(\mathrm{A})$ and $(\mathrm{C})$ after the intermediate portion.

- The mean value of these two time values is defined as the reference point to split the data.

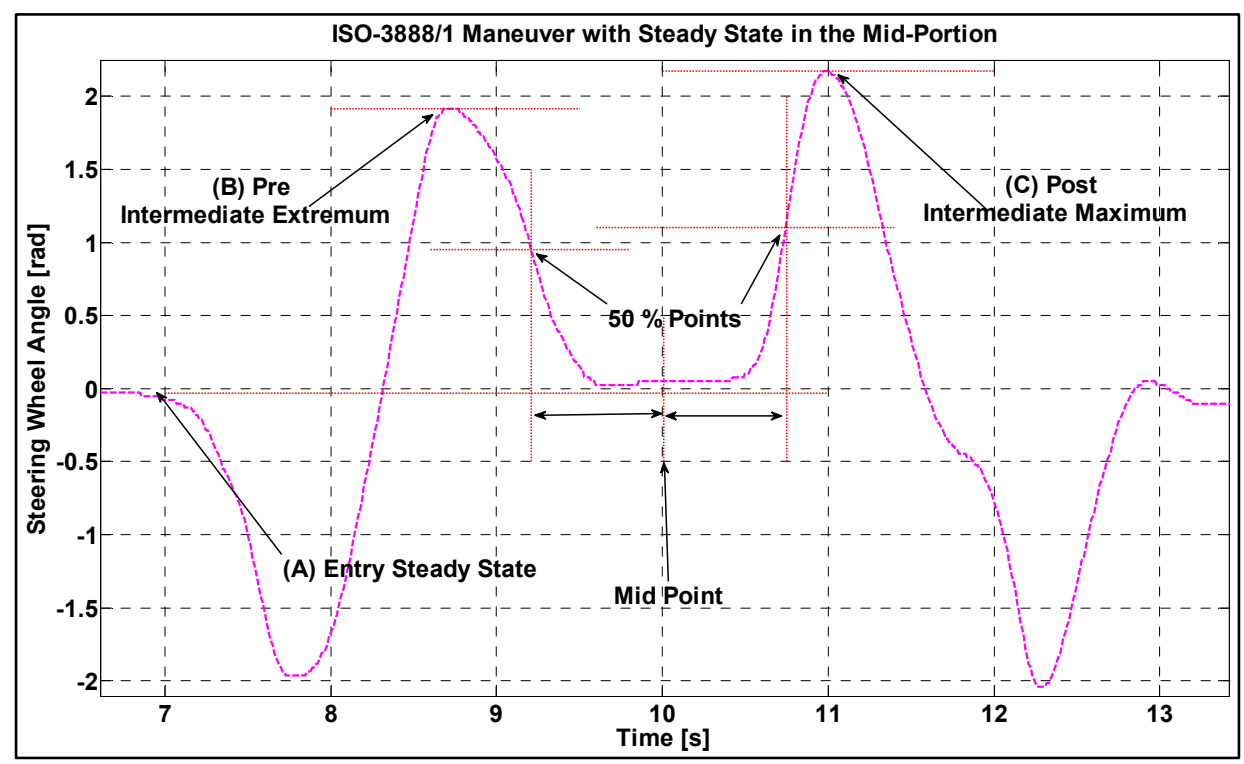

Figure 5: Definition of the reference point for data splitting

Such a definition of reference value makes use of the experimental independent characteristic values and is more robust than the previously mentioned techniques.

Once the reference point is defined to split the data the following metrics can be defined:

- Overall lag

- Lags, and ratio of the lags for two portions of the data

- Ratios of maximum outputs and inputs, and the ratio of ratios.

\subsection{Data Alignment}

The question to be examined in this section is, when comparing the results of a closed loop maneuver with the results of a simulation model, is it enough to define and calculate metrics for each test run; or is it needed to devise a methodology to align the time histories of the experimental data?

Aligning the data is not needed to define and calculate performance metrics for separate test cases, but aligning the outputs of different test runs can be utilized to statistically generate an experimental data zone by calculating the mean values and standard deviations (and thus the confidence intervals) of different test runs at each time step. This experimental data zone can be used to check if the time histories of the simulation outputs remain inside them, which is previously proposed as a validity criterion (Heydinger 1990).

In order to align the data, a reference point is needed for each part of the split data. For example, in a step response experiment, the time point at which the steering wheel angle reaches $50 \%$ of its final value 


\section{Kutluay and Winner}

can be used to define relevant time domain performance metrics and to align different test results (ISO 2003). In the double lane change maneuver, the steering wheel angle does not reach the steady state except at the start and the end of the maneuver (although a steady state can possibly exist in the middle portion depending on the test conditions, i.e. low speed, different track dimensions, different vehicle).

As mentioned above, a midpoint needs to be defined in order to split the data for cross-correlation analysis. Thus, instead of seeking a general reference point to align the whole time history, it is more logical to find two reference points for the former and latter portions of the data and analyze these portions separately.

This reference points can be defined as:

a. Time at which the steering wheel angle reaches $50 \%$ of its first maximum value for the first portion, and mean value between the entry steady state value and the first extremum after the midpoint for the second portion (Figure 6). In the first portion, this point is between the steady state value attained at the entrance area of the track and the first maximum of the steering wheel angle. In the second portion, this point is between end of the transition region and the consequent extremum of the steering wheel angle that is attained as the vehicle leaves the middle lane towards the exit area of the track. This approach is similar to the alignment method of the step input maneuver (ISO 2003).

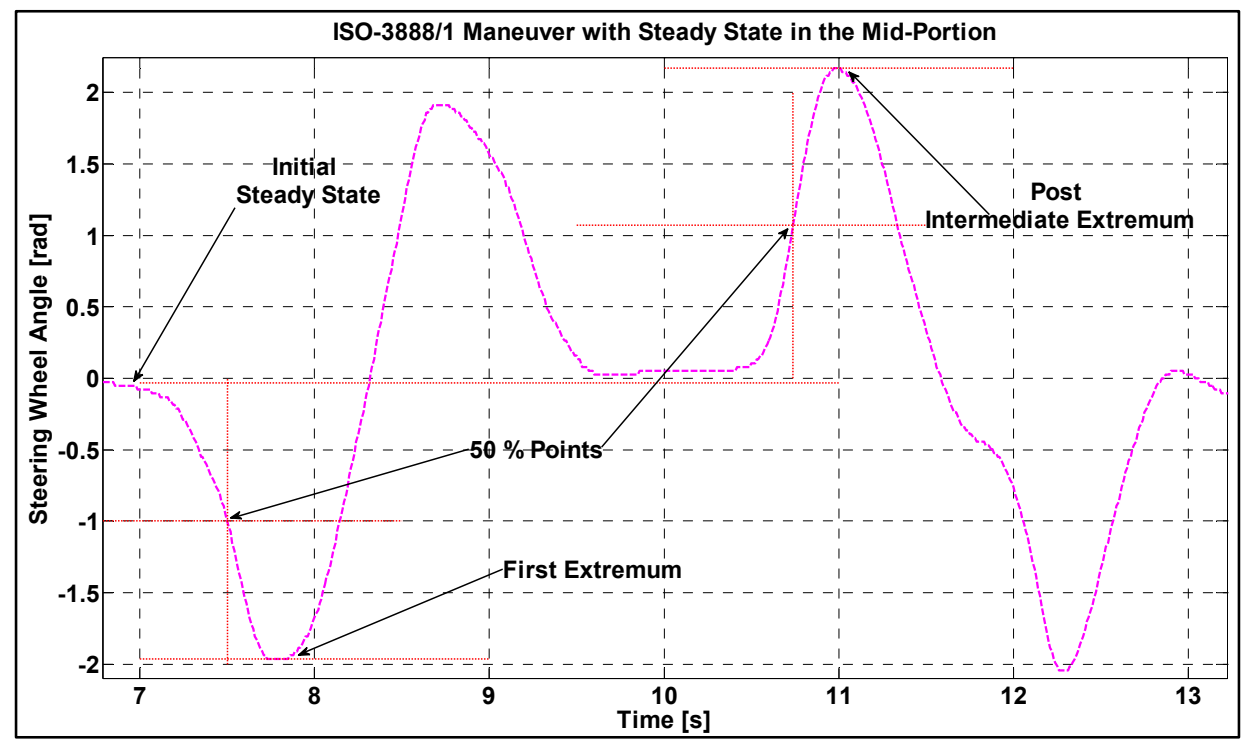

Figure 6: Definition of reference points according to (a)

b. Time at which the steering wheel angle reaches the mean value of the first and second extrema for the first portion, and the last and second to last extrema for the second portion. In this case, different from (a), the metrics should be defined using the time differences on either side of the reference points (Figure 7).

Once the data alignment is completed, confidence intervals and other performance metrics, such as the time coordinates of the maximums with respect to the reference times can be defined.

It should be noted that, another, and considerably simpler, approach would be, instead of taking two separate portions to define the experimental data zone, simply use the first reference point defined in (a) in order to analyze the whole time history.

The opinion of the author of this document is that the maneuver has two distinct portions. The first portion of the maneuver is independent of the second portion, and the second portion of the maneuver is not a natural extension of the first maneuver, like the second half period of a sine, but a consecutive and 


\section{Kutluay and Winner}

similar maneuver with its own characteristics. Because of these properties, the maneuver should be analyzed in two portions.

It should also be noted that, the two portions are connected in the manner that the second one is executed immediately after the first one and because of this; the initial conditions of two portions are different. The resulting dynamics from the first portion propagate into the second portion, whereas the first portion starts from a steady state straight line driving condition.

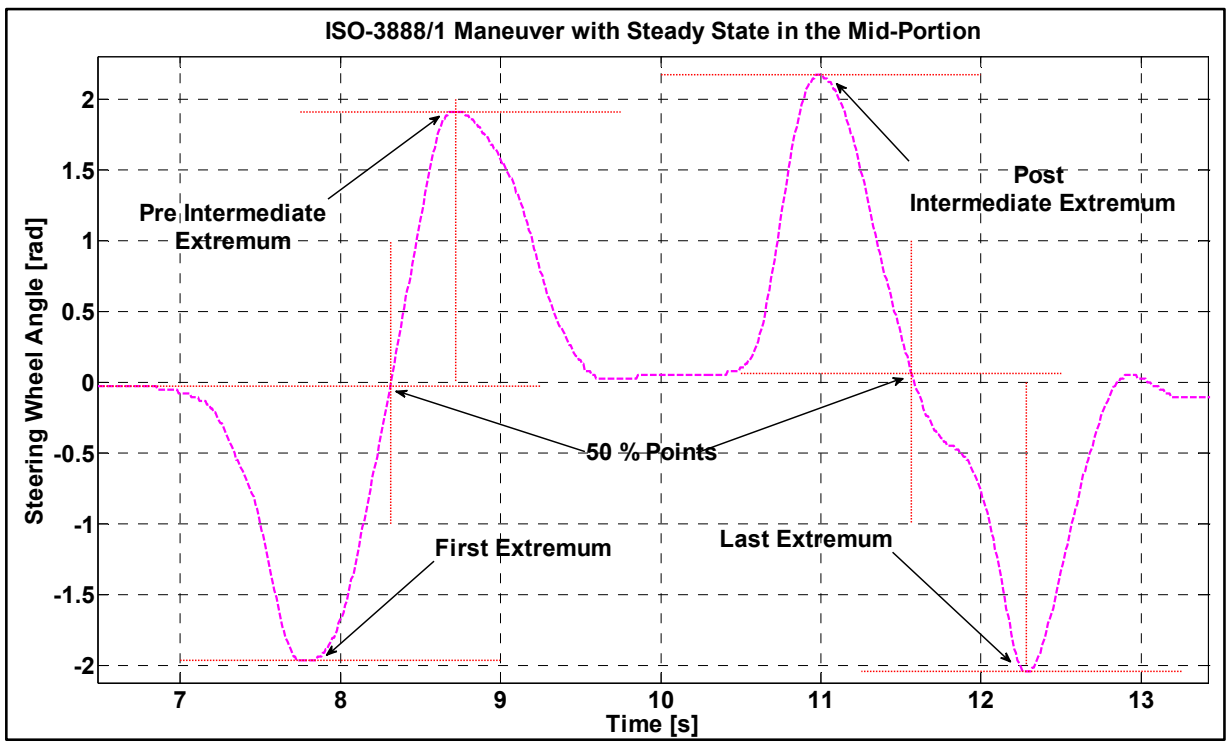

Figure 7: Definition of reference points according to (b)

\section{DATA HANDLING METHODOLOGY}

In this section the methodologies to handle the experimental and simulation data are presented. Once "enough" number of test maneuvers are performed and experimental data are collected, the recorded input time history is used to run the simulations. The response of the real system as well as the output of the simulations need to be handled, i.e. split and aligned, in order to be able to perform further statistical analysis.

\subsection{Experimental Data}

The techniques to split and align the data and the possible metrics to be regarded are presented in the second chapter. The proposed methodology, Figure 8 , can be summarized as:

- Calculating the overall lag

- Defining the midpoint and splitting the data

- Lags, and ratio of the lags for two portions of the data

- Ratios of maximum outputs and inputs, and the ratio of ratios

- Defining reference points for data alignment

- Calculating other metrics, average outputs and confidence 


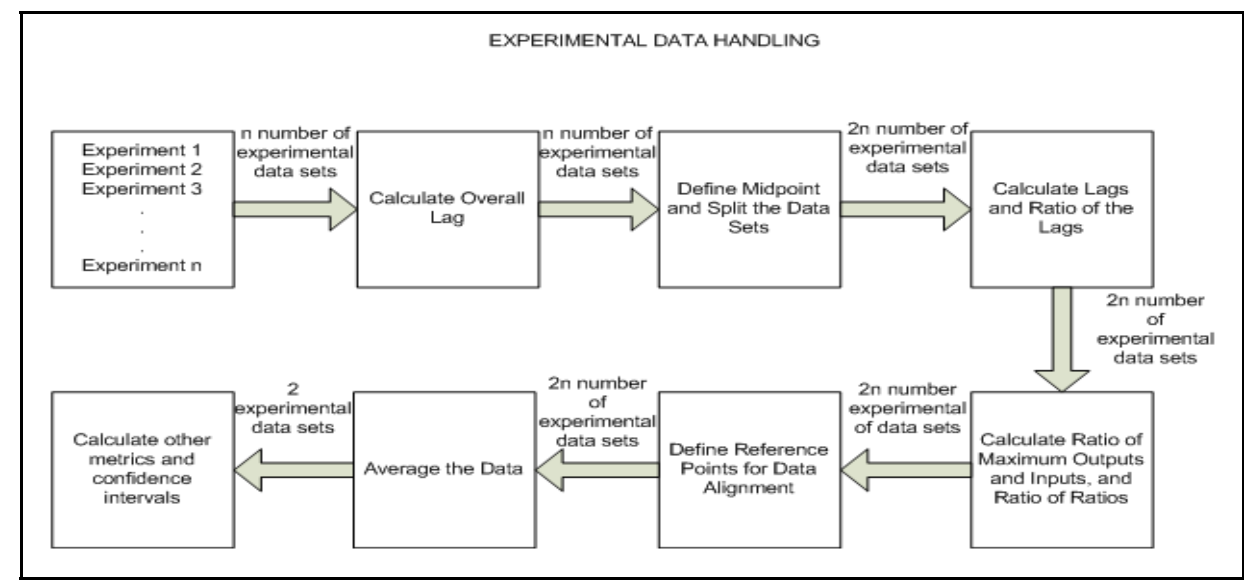

Figure 8: Flow diagram of the experimental data handling method

\subsection{Simulation Data}

The simulations are run using the experimentally measured input data. The start and end conditions of the input data is defined using the steady state criterion. Each experimental run is analyzed using a previously written MATLAB ${ }^{\circledR}$ function to find out the regions in which the signal is steady state.

In simulation data handling, there are three possible paths to follow, depending on if the experimental inputs are first reduced to an averaged simulation input and if the data analysis is performed using one interval, or two intervals. A summary of these paths are shown in Figure 9.

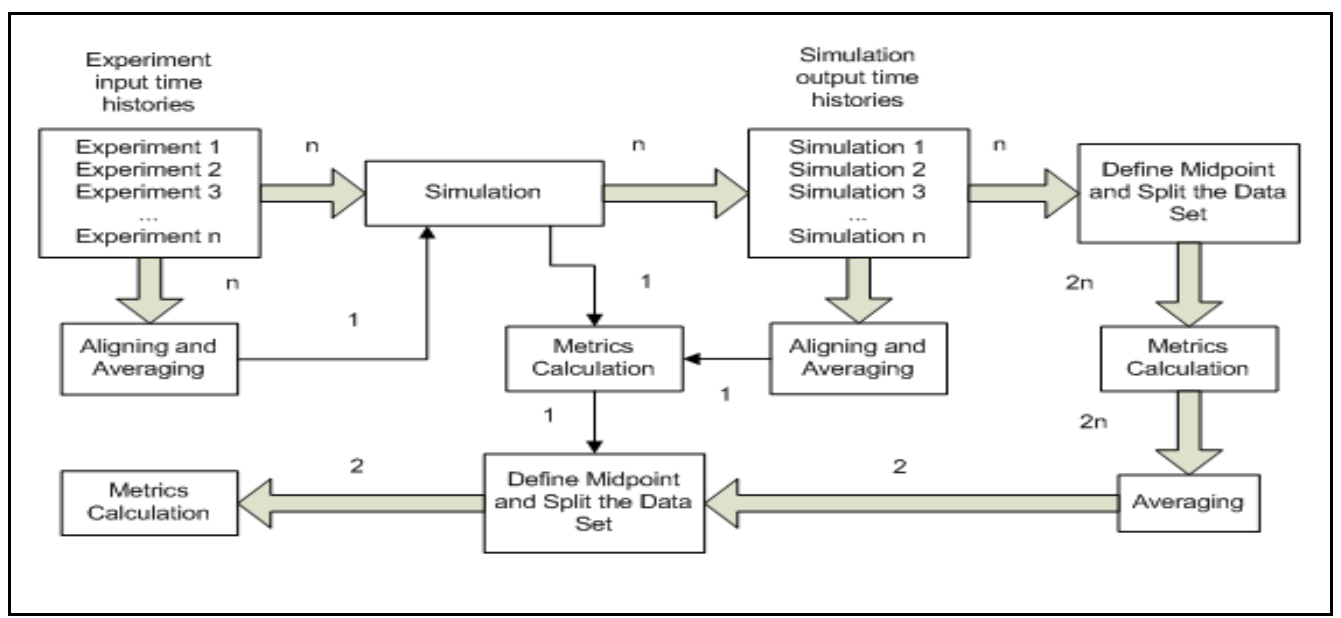

Figure 9: Summary of simulation data handling methods

\subsubsection{Averaged Input Case}

The experimental inputs can be aligned and averaged to obtain an average input time history which yields one simulation time history. This simulation time history is not the average of individual simulations, but the result of a simulation run by using the average time history of the measured input signals. The order of operations in this case is:

- Aligning and averaging the inputs and running the simulation

- Calculating overall lag and other metrics

- Defining the midpoint and splitting the data 


\section{Kutluay and Winner}

- Calculating lags, and ratio of the lags for two portions of the data

- Ratios of maximum outputs and inputs, and the ratio of ratios

Figure 10 shows the flow diagram of for this case. This method is appropriate for complex simulation models for which performing only one simulation is more feasible than processing each experimental input one at a time. The other option is running simulations separately for each experimental measurement and then handling the data. These options are explored in the following two sections.

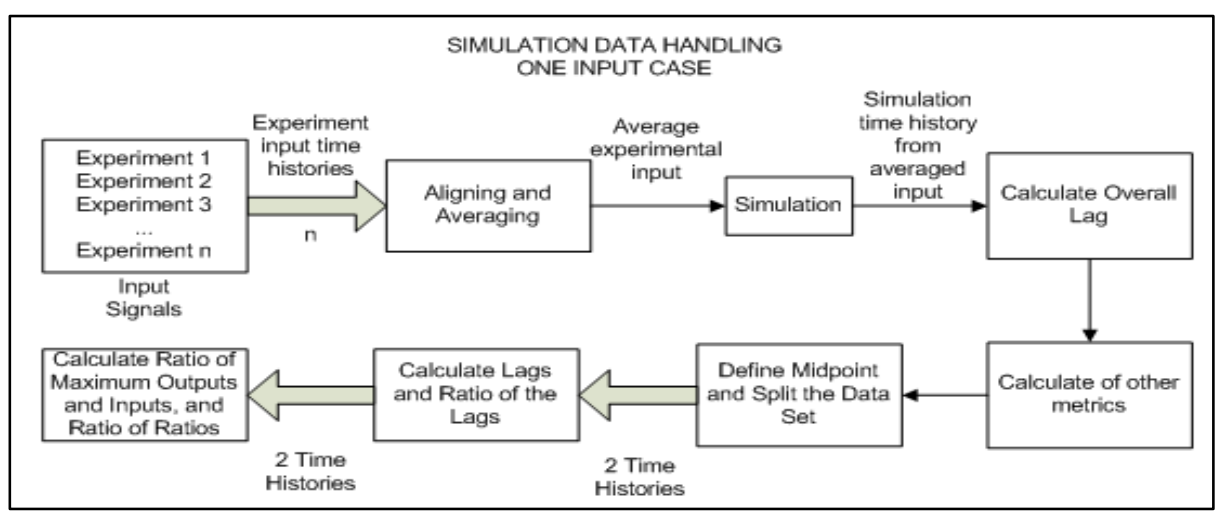

Figure 10: Simulation data handling method for averaged input case

\subsubsection{Averaged Output One Interval Case}

This case, demonstrated in Figure 11) explores the possibility to perform a simulation for each maneuver measurement and then averaging the outputs and assessing the metrics using one reference point for alignment.

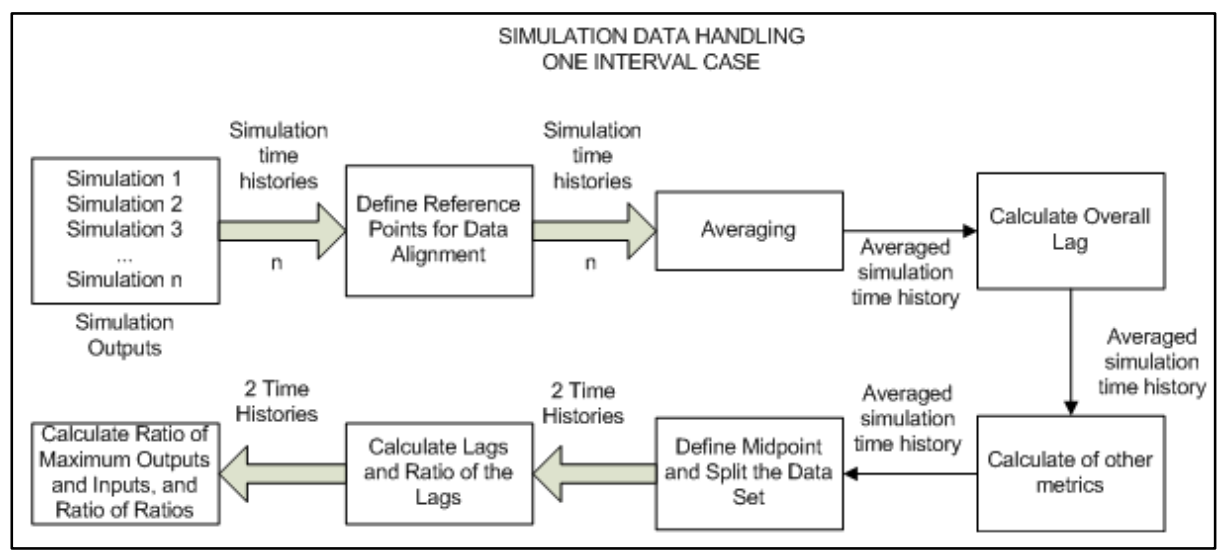

Figure 11: Simulation data handling method for averaged output one interval case

Steps in this case are:

- Defining the reference point for each data set

- Averaging the data sets

- Calculating overall lag and other metrics

- Defining the midpoint and splitting the data

- Calculating lags, and ratio of the lags for two portions of the data

- Ratios of maximum outputs and inputs, and the ratio of ratios 


\section{Kutluay and Winner}

\subsubsection{Averaged Output Two Intervals Case}

In this case the simulation is performed for each maneuver measurement and then the outputs are averaged. Assessment of the metrics is done using two reference point for alignment. The proposed methodology, Figure 12, can be summarized as::

- Calculating the overall lag for each case

- Defining the midpoint and splitting the data for each data set

- Calculating Lags, and ratio of the lags for two portions of each of the data sets

- Ratios of maximum outputs and inputs, and the ratio of ratios of each of the data sets and then averaging

- Defining the reference points for data alignment and averaging

- Lags, and ratio of the lags for two portions (optional)

- Ratios of maximum outputs and inputs, and the ratio of ratios (optional)

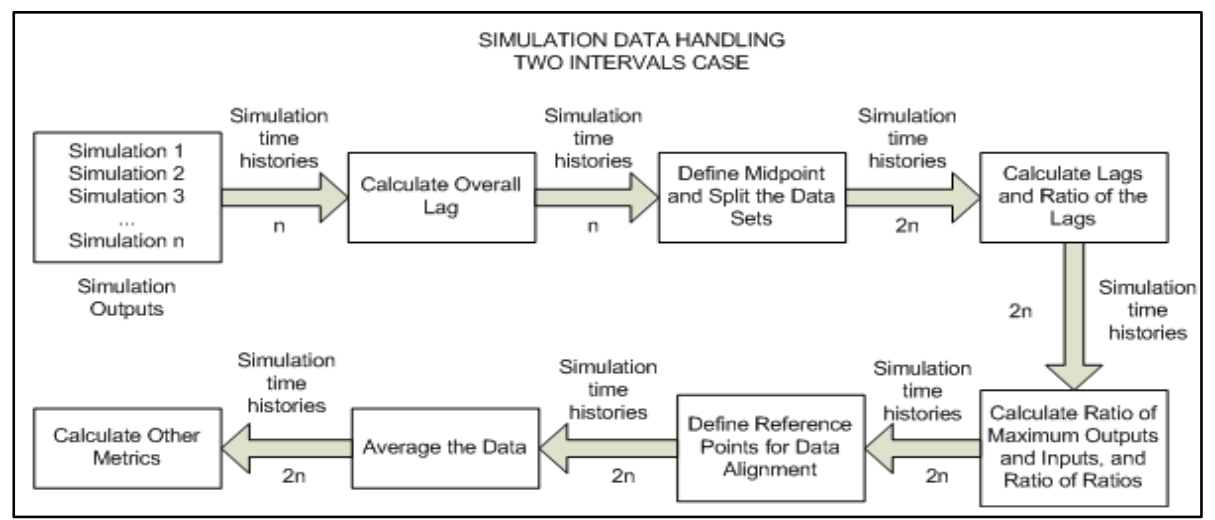

Figure 12: Simulation data handling method for averaged output two intervals case

The first of these methods should only be followed if the simulation model is very complex or running separate simulations for each experiment is not feasible due to required workload, time or costs. Of the latter two methods, which are compared in Figure 13, the two interval method captures the two distinct portions of the maneuver and provides more comparable and better defined metrics. Table 1 presents the average of measured steering wheel angle magnitudes and temporal coordinates at the third and the fourth extrema for the data sets shown in Figure 13.

Table 1: Temporal and spatial coordinates of the third and fourth extrema, calculated with the one and two interval approaches

\begin{tabular}{|c|c|c|c|c|}
\hline \multirow{2}{*}{$\begin{array}{c}\text { Average Steering } \\
\text { Wheel Angle }\end{array}$} & \multicolumn{2}{|c|}{ Third Extremum } & \multicolumn{2}{c|}{ Fourth Extremum } \\
\cline { 2 - 5 } & One Interval & $\begin{array}{c}\text { Two Inter- } \\
\text { vals }\end{array}$ & One Interval & $\begin{array}{c}\text { Two Inter- } \\
\text { vals }\end{array}$ \\
\hline Time [s] & 0.2 & 0.3 & 1.35 & 1.55 \\
\hline Amplitude [rad] & 2.086 & 2.237 & -1.689 & -1.862 \\
\hline
\end{tabular}

When Figure 13 is examined, it is seen that the lowest steering wheel angle amplitude is 2.15 radians for the third extremum, and -1.75 radians for the fourth extremum. Comparing these figures with Table 1, it is clear that one interval approach performs poorly in aligning the input signal in the second part of the maneuver. Averaged steering wheel angle signals for one interval and two intervals cases are presented in Figure 14. 
Kutluay and Winner

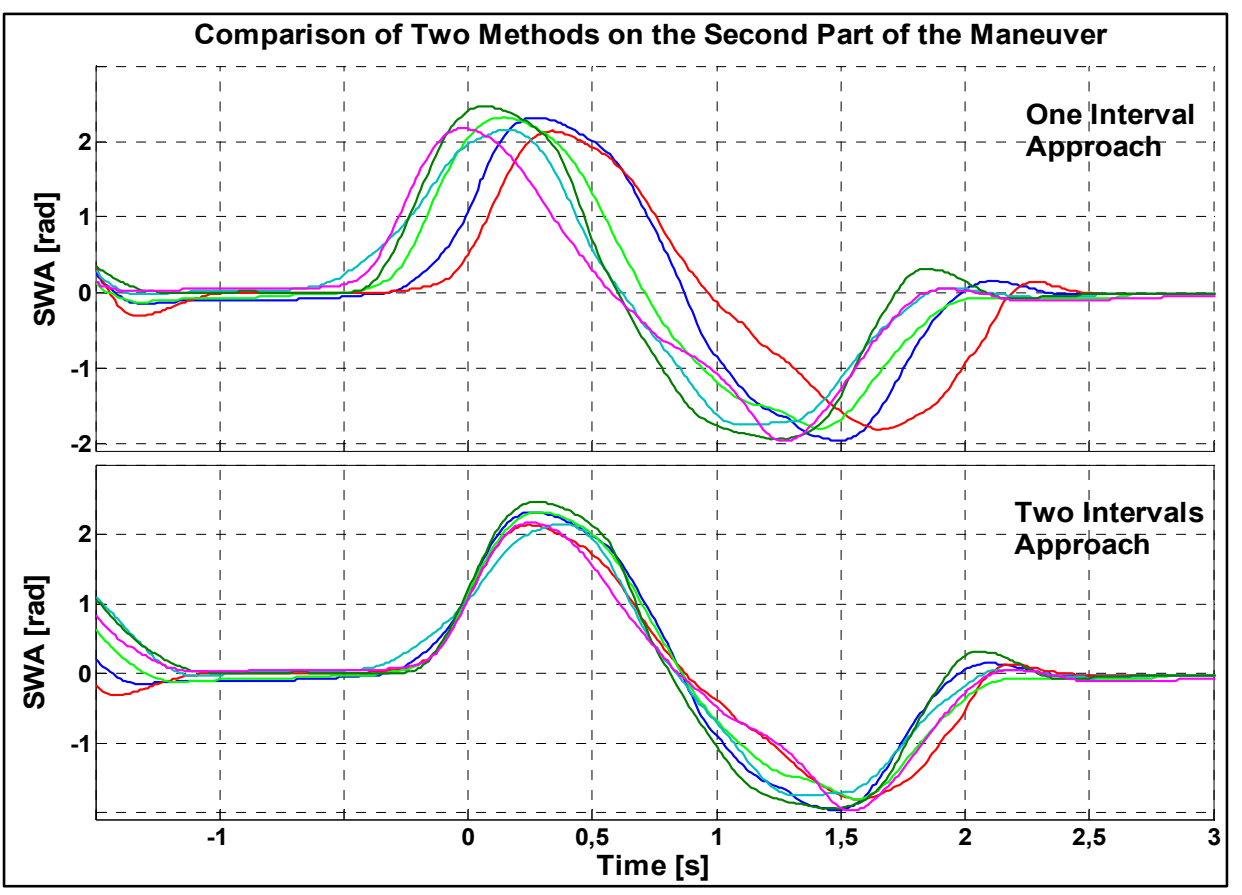

Figure 13: Comparison of one interval and two intervals approaches

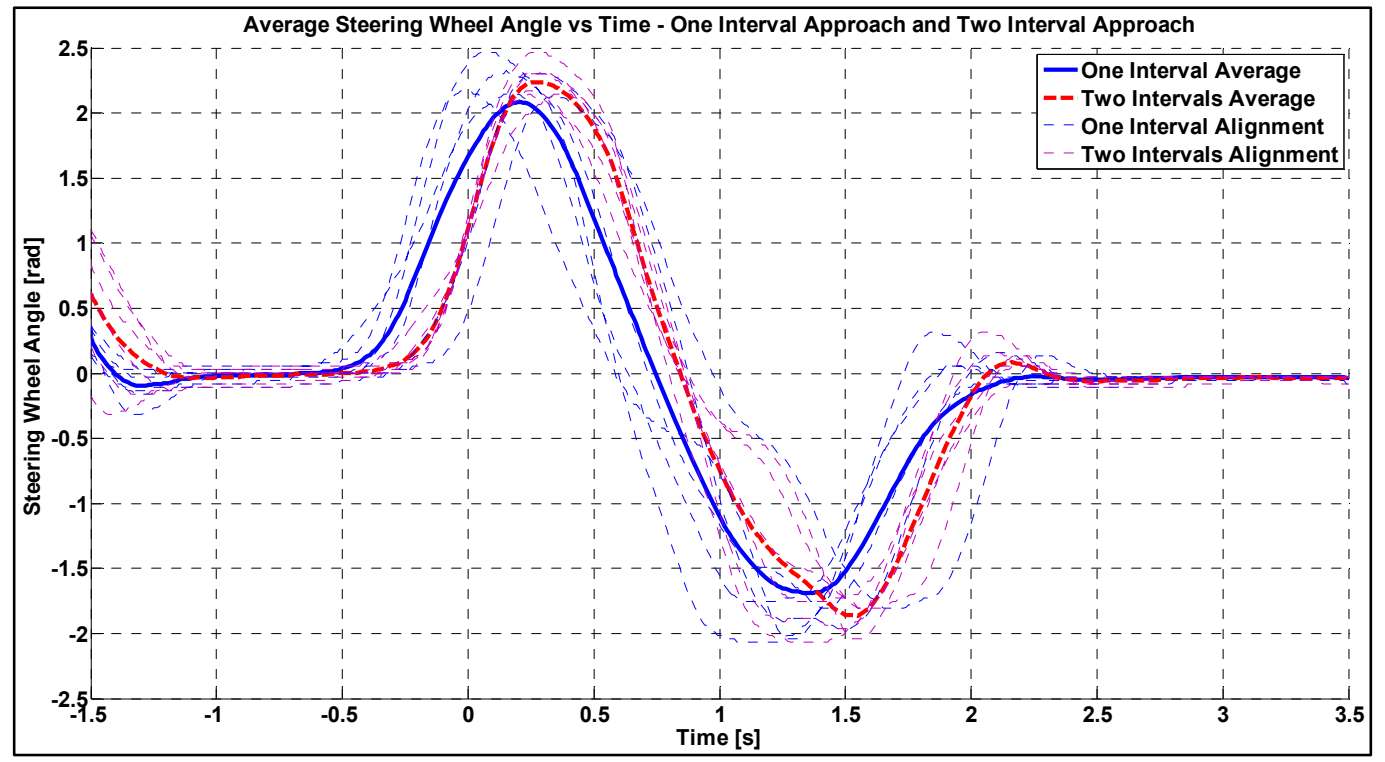

Figure 14: Average Steering Wheel Angles

\section{CONCLUSION}

This document presents a methodology to assess the experimental and simulated data of the ISO-3888/1 double lane change maneuver for validation of validation dynamics simulation models. Different approaches to assess the data and define the reference points are presented. Possible methods to define the analysis intervals and determine the reference points are explained and discussed. A method to process the experimental data and three methods to process the simulation data are introduced. 


\section{Kutluay and Winner}

As mentioned above, the opinion of the authors is that the maneuver has two distinct portions. In the one portion approach, the discrepancy between different experiments tends to be higher than the two portion approach in the second part of the maneuver, as clearly demonstrated in Figure 13. Moreover one interval approach is shown to yield lower average amplitudes in the extrema than the original signals. Therefore it is more appropriate to use the approach with two portions which enables the analyst to isolate and assess the second part of the maneuver more effectively, although it has slightly more workload than its alternative, the single interval approach.

\section{ACKNOWLEDGMENTS}

The experimental results used in the diagrams are obtained in test drives using an Opel Astra $\mathrm{H}$ as test vehicle. All collected data are from the measurements of the sensor of the ESC system of the vehicle and are recorded through the CAN-Bus. Data processing is performed in MATLAB ${ }^{\circledR}$. Students Aziz Çağlar Alaloğlu, Halil İbrahim Özel and Mustafa Hakan Sandık are hereby acknowledged for their contributions.

\section{REFERENCES}

Allen, R.W., T. J. Rosenthal, D. H. Klyde, K. J. Owens, and H. T. Szostak. 1992. "Validation of Ground Vehicle Computer Simulations Developed for Dynamics Stability Analysis“, SAE-920054

Braghin, F., F. Cheli, S. Melzi, and F. Resta. 2006. "Tyre Wear Model: Validation and Sensitivity Analysis“, Meccanica (2006) 41:143-156, Springer

Heydinger, G. J., W. R. Garrott, J. P. Chrstos, and D. A. Guenther. 1990. "A Methodology for Validating Vehicle Dynamics Simulations", SAE-900128

ISO 3888-1. 1999. "Passenger cars - Test track for a severe lane change manoeuvre - Part 1: Double lane-change"

ISO 7401. 2003. "Road vehicles - Lateral transient response test methods - Open-loop test methods"

ISO 4138. 2004. "Passenger cars - Steady-state circular driving behaviour - Open-loop test methods"

ISO 7975. 2006. "Passenger cars - Braking in a turn - Open-loop test method"

Draft Proposal for an International Standart, Road Vehicle -Transient Response Test Procedures ISO/TC22/SC9/N194. 1979.

Ozan, B., P. Sendur, M. E. Uyanik, Y. Oz, and S. I. Yilmaz. 2010. "A Model Validation Methodology for Evaluating Rollover Resistance Performance of a Ford Commercial Vehicle“, SAE-2010-01-0107

Winkler, C. B., R. L. Nisonger and R. D. Ervin. 1978. "Testing the Michigan Double-Bottom Tanker", SAE-781066

\section{AUTHOR BIOGRAPHIES}

EMIR KUTLUAY studied mechanical engineering at the Middle East Technical University. From 2007 to 2012 he worked on his Ph.D. at the Institute of Automotive Engineering (FZD) in TU Darmstadt, studying validation methods for vehicle dynamics simulations. His email address is emir.kutluay@googlemail.com.

HERMANN WINNER is professor in the Mechanical Department of TU Darmstadt, and head of the Institute of Automotive Engineering (FZD). His email address is winner@fzd.tu-darmstadt.de. 